\title{
Minimally Invasive Mitral Valve Surgery III Training and Robotic-Assisted Approaches
}

\author{
Eric J. Lehr, MD, PhD, * T. Sloane Guy, MD, † Robert L. Smith, MD, $\neq$ Eugene A. Grossi, MD, $\S$ \\ Richard J. Shemin, MD,// Evelio Rodriguez, MD, $\mid$ Gorav Ailawadi, MD,\# Arvind K. Agnihotri, MD, ** \\ Trevor M. Fayers, FRACS, †† W. Clark Hargrove, MD, $\neq$ Brian W. Hummel, MD, $\oint \oint$ \\ Junaid H. Khan, MD,//// S. Chris Malaisrie, MD, 19 John R. Mehall, MD, \#\# Douglas A. Murphy, MD, *** \\ William H. Ryan, MD, $\neq$ Arash Salemi, MD, †† Romualdo J. Segurola, Jr, MD, $\neq \neq$ \\ J. Michael Smith, MD, $\oint \oint$ J. Alan Wolfe, MD,///// Paul W. Weldner, MD, 1999 Glenn R. Barnhart, MD, * \\ Scott M. Goldman, MD,\#\#\# and Clifton T. P. Lewis, MD****
}

\begin{abstract}
Minimally invasive mitral valve operations are increasingly common in the United States, but robotic-assisted approaches have not been widely adopted for a variety of reasons. This expert opinion reviews the state of the art and defines best practices, training, and techniques for developing a successful robotics program.
\end{abstract}

Accepted for publication June 16, 2016

From the *Swedish Heart and Vascular Institute, Seattle, WA USA; †Temple University, Philadelphia, PA USA; \$The Heart Hospital Baylor Plano, Plano, TX USA; §New York University School of Medicine, New York, NY USA; ||David Geffen School of Medicine, University of California Los Angeles, Los Angeles, CA USA; - STt. Thomas Hospital, Nashville, TN USA; \#University of Virginia, Charlottesville, VA USA; **Saint Elizabeth's Medical Center, Brighton, MA USA; ††Holy Spirit Northside Hospital, Chermside, Australia; \$†Penn Presbyterian Medical Center, Philadelphia, PA USA; §§Gulf Coast Cardiothoracic \& Vascular Surgeons, Ft. Myers, FL USA; |||East Bay Cardiac Surgery Center, Oakland, CA USA; बINorthwestern University, Feinberg School of Medicine, Chicago, IL USA; \#\#Penrose St. Francis Hospital, Colorado Springs, CO USA; ***Emory St. Joseph's Hospital, Atlanta, GA USA; †+Weill Cornel Medical College/New York Presbyterian Hospital, New York, NY USA; t+South Florida Heart \& Lung Institute, Doral, FL USA; $\S \S \S$ TriHealth Heart Institute, Cincinnati, OH USA; ||||||Northeast Georgia Physicians Group, Gainesville, GA USA; $\$ \uparrow$ Central Maine Heart and Vascular Institute, Lewiston, ME USA; \#\#\#Lankenau Medical Center, Wynnewood, PA USA; and ****Department of Cardiothoracic Surgery, Princeton Baptist Hospital, Birmingham, AL USA.

Supported by Princeton Baptist Hospital, Birmingham, AL USA, which provided funds for medical editing assistance, which was performed by Jeanne McAdara-Berkowitz, $\mathrm{PhD}$. The funds came from an educational budget and were not provided by any commercial entity. Medical illustrations created by Jill Rhead MA, CMI, FAMI were donated by Edwards Lifesciences Corp, Irvine, CA USA

Disclosures: See next page.

Address correspondence and reprint requests to Clifton T. P. Lewis, MD, Princeton Baptist Medical Center, 833 Princeton Ave, SW, POB III, Suite 200A, Birmingham, AL 35211 USA. E-mail: cliftonlewis1942@gmail.com.

Copyright (C) 2016 by the International Society for Minimally Invasive Cardiothoracic Surgery

This is an open-access article distributed under the terms of the Creative Commons Attribution-Non Commercial-No Derivatives License 4.0 (CCBY-NC-ND), where it is permissible to download and share the work provided it is properly cited. The work cannot be changed in any way or used commercially.

ISSN: $1556-9845 / 16 / 1104-0260$
Key Words: Minimally invasive surgery (includes port access, minithoracotomy), Mitral valve, repair, replacement, Surgery/incisions/ exposure/techniques, MVR, MIMVR, Heart valve, Robotic surgery.

(Innovations 2016;11: 260-267)

n 1998, Mohr et al and Carpentier et al independently performed the first 2 robotic-assisted, minimally invasive mitral valve (MV) repairs using the da Vinci robotic surgical platform (Intuitive Surgical, Inc, Sunnyvale, CA USA), ${ }^{1,2}$ followed closely by Chitwood et al. ${ }^{3,4}$ The da Vinci platform was approved for robotic-assisted, minimally invasive MV repair and replacement (MIMVR) by the US Food and Drug Administration in 2002, in part based on a multicenter, phase 2 clinical trial, the results of which were reported in 2005 by Nifong et al. ${ }^{5}$

\section{See accompanying editorial on page 233 , and articles on pages 243 and 251}

Since approval, multiple investigators have reported good to excellent results in large series of patients undergoing roboticassisted MIMVR. ${ }^{5-12}$ Ongoing areas of investigation include patient selection, training robotic-surgery teams, the inclusion of concurrent procedures, and standardization of technique. To provide guidance on these issues, 26 surgeons whose combined experience includes approximately 17,000 MIMVR operations formed a working group to define a consensus-based, bestpractices approach for the standardization of technique, patient selection, and team training for traditional and robotic-assisted MIMVR. Of these 26 surgeons, 11 have significant experience with robotic techniques ( $\geq 50$ career cases, up to $>1,500$ cases), and another 4 surgeons have more limited but increasing experience. The remaining surgeons who do not perform robotic techniques nevertheless provided an indispensable source of critical discussion as this paper was being developed.

This article is the third of a 3-part series and focuses on considerations specific to robotic-assisted MIMVR, including training pathways that will prepare the surgical team to move from sternotomy to port access to robotic approaches. The 3 papers are intended to be read together; patient screening and selection, and preoperative planning for successful cannulation, 
Disclosures: All authors declare that they serve as consultants on the Medica Advisory Board for Edwards Lifesciences Corp, Irvine, CA USA. In addition, T. Sloane Guy, MD, is a consultant to Medtronic Inc, Minneapolis, MN USA, and Ethicon Inc/Johnson\&Johnson, Somerville, NJ USA, and a recipient of a grant from Biomet, Inc, Warsaw, IN USA. Robert L. Smith, MD, serves on the speaker's bureaus for Abbott Vascular, Abbott Park, IL USA, and Intuitive Surgical, Inc, Sunnyvale, CA USA. Eugene A. Grossi, $\mathrm{MD}$, is a consultant to Medtronic, Inc, Minneapolis, MN USA, and Intuitive Surgical, Sunnyvale, CA USA. Evelio Rodriguez, MD, is a consultant to Abbott Vascular, Abbott Park, IL USA, AtriCure, Inc, West Chester, OH USA and St. Jude Medical, Inc, St. Paul, MN USA, is a grant recipient from Medtronic, Inc, Minneapolis, MN USA, and serves on the speaker's bureaus for Edwards Lifesciences, Corp, Irvine, CA USA, and Medtronic, Inc, Minneapolis, MN USA. Gorav Ailawadi, MD, is a consultant to Abbot Vascular, Abbott Park, IL USA, AtriCure, Inc, West Chester, OH USA, Medtronic, Inc, Minneapolis, MN USA, and St. Jude Medical, Inc, St. Paul, MN USA. W. Clark Hargrove, MD, was a consultant to Sorin/LivaNova, Milan, Italy. Brian W. Hummel, MD, is a consultant to AtriCure, Inc, West Chester, OH USA, and Medtronic, Inc, Minneapolis, MN USA. S. Chris Malaisrie, MD, is a consultant for Medtronic, Inc, Minneapolis, MN USA and Abbott Vascular, Abbott Park, IL USA. John R. Mehall, MD, is a consultant to AtriCure, Inc, West Chester, OH USA. Douglas A. Murphy, MD, receives product royalties from Medtronic, Inc, Minneapolis, MN USA William H. Ryan, MD, is a consultant to Medtronic, Inc, Minneapolis, MN USA. Arash Salemi, MD, is a consultant to Medtronic, Inc, Minneapolis, MN USA. J. Michael Smith, MD, is a consultant to AtriCure, Inc, West Chester, OH USA, and Intuitive Surgical, Sunnyvale, CA USA. Glenn R. Barnhart, MD, is a consultant to AtriCure, Inc, West Chester, OH USA, and On-X Life Technologies, Austin, TX USA. Scott M. Goldman, MD, serves on advisory boards for Abbott Vascular, Abbott Park, IL USA, and Medtronic, Inc, Minneapolis, MN USA, and is a consultant to St. Jude, Inc, St. Paul, MN USA, and LSI SOLUTIONS, Victor, NY USA. Clifton T. P. Lewis, MD, is a consultant to Intuitive Surgical, Inc, Sunnyvale, CA USA. Eric J. Lehr MD, PhD, Richard J. Shemin MD, Arvind K. Agnihotri MD, Trevor M. Fayers, MD, Junaid H. Khan MD, Romualdo J. Segurola, Jr, MD, J. Alan Wolfe, MD, and Paul W. Weldner, MD, declare no other conflicts of interest.

systemic perfusion, myocardial protection, and anesthetic preparation, were treated in the first part of the series, ${ }^{13}$ whereas the second report described intraoperative techniques to perform nonrobotic MIMVR while minimizing complications. ${ }^{14}$

\section{PATIENT SELECTION}

The suitability of a patient for robotic-assisted MIMVR depends on chest anatomy and status of the vasculature. General guidelines for patient selection are found in Ailawadi et al, ${ }^{13}$ with considerations specific to robotic approaches discussed here. The ideal patient is tall, thin, and without obstruction in the right hemithorax; this body habitus provides generous intrathoracic workspace once the right lung is collapsed and permits easy triangulation of the camera and instruments. Departure from this "ideal" introduces additional challenges that may be surmountable with surgeon's experience and appropriate modification of technique.

Obese patients need longer instrument ports and a more generous working port, often supported with an extra small soft tissue retractor. Instrument exchanges can be more difficult. Large breasts are retracted medially to enhance exposure of the lateral chest wall.

An elevated hemidiaphragm can obstruct visualization and make passage of right-arm instruments more difficult. Solutions to avoid diaphragmatic injury include reverse-Trendelenburg position, a diaphragmatic retention suture, and a bariatric port for the right instrument arm and resection of the intrathoracic pericardial fat pad.

Anatomical challenges and prior right thoracic procedures, including pleurodesis, chest tubes, and pulmonary resections or pleurisy, may result in moderate adhesions that can be lysed but may require a larger working port for access. More severe adhesions are a contraindication for robotic-assisted MIMVR and may require conversion to sternotomy. Severe pectus excavatum and kyphoscoliosis displace the heart leftward, alter the position of the heart relative to the intercostal spaces, and dramatically reduce the working space. In such patients, the mitral annulus may lie anteriorly, close to the posterior table of the sternum, hampering visualization of the MV apparatus. Exposure may be facilitated by more posterolateral placement of the camera and working ports.

Because retrograde arterial perfusion is the mainstay of a totally endoscopic approach, atypical vascular anatomy must be considered. ${ }^{15}$ Preoperative computed tomography angiography can identify calcific and noncalcific atheroma, tortuosity, localized dissections, and aberrant and small vessels, allowing the surgeon to select an alternate cannulation strategy or conventional sternotomy, avoiding the potential complications of retrograde arterial perfusion.

Previous cardiac surgery is not a contraindication to a robotic approach and may actually avoid many of the complexities of a subsequent sternotomy. However, redo procedures should be avoided until the team has considerable experience. Special considerations for myocardial preservation, including systemic cooling, should be made in cases with patent internal mammary arterial grafts.

\section{SURGICAL TECHNIQUE}

\section{Anesthesia and Monitoring}

Dedicated support from the anesthesia team with expertise in transesophageal echocardiography (TEE) is crucial, as they will actively participate in cannulation and line placement.

Single lung ventilation simplifies port placement and can be accomplished with either a double lumen endotracheal tube or a single lumen tube and bronchial blocker. The choice of monitoring lines used is directed by the cannulation, cardiopulmonary bypass, aortic cross-clamping, and myocardial protection strategies, which are discussed in detail in other portions of this series. ${ }^{13,14}$

When cardioplegia is delivered through the chest, necklines may be identical to those used with sternal operations and can be determined by local custom and surgeon's preference. A percutaneous pulmonary artery vent may augment venous drainage, particularly in large patients.

\section{Patient Positioning}

The patient is placed on the operating table in supine position, with the right hemithorax elevated 30 degrees and the hips flat (Fig. 1). Defibrillator pads are applied outside the operative field but with consideration for sternotomy should it become necessary. The arms are tucked, with the right shoulder slightly extended and the elbow flexed. The right arm hangs supported by a sheet below the operating table, giving access to the right axilla for the transthoracic cross-clamp and to exteriorize the pericardial retraction sutures more posteriorly. When positioning the patient, consideration should be given to avoid collision of the robotic arms with the patient's head, endotracheal tube, abdomen, and pelvis.

\section{Cannulation}

The common femoral artery is the most common site for perfusion in robotic-assisted cardiac surgery. Either side is 


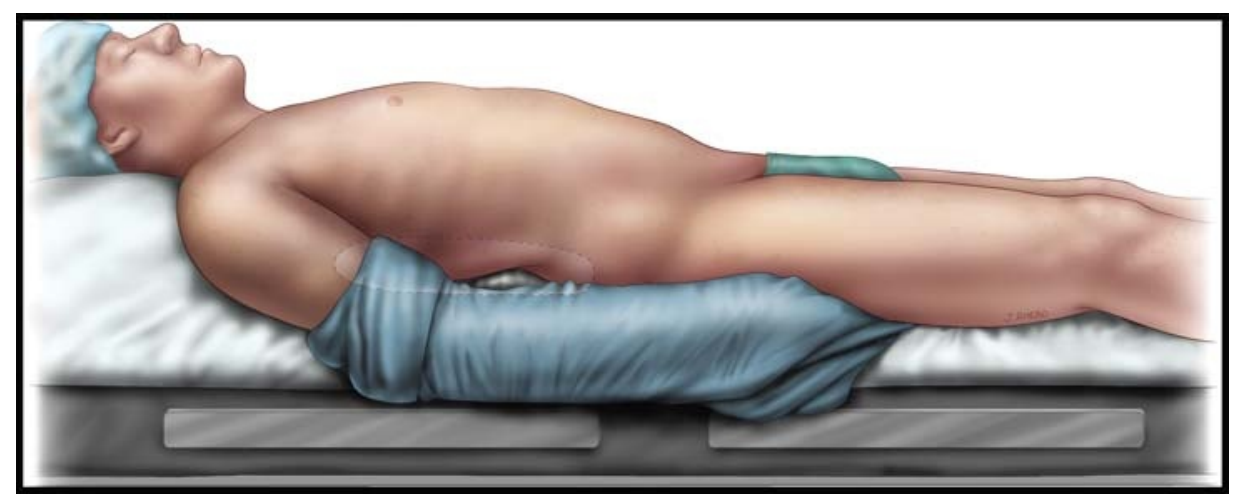

FIGURE 1. Patient positioning for robotic-assisted MIMVR showing a small pillow placed inferior to the scapula (transparent ellipse).

appropriate, although the right side is more convenient because it is more accessible when the robot is docked.

Direct aortic cannulation is possible in robotic MV procedures when peripheral vascular disease prevents a femoral ap$\operatorname{proach}^{16}$; however, there are several specific limitations for robotic-assisted procedures. This approach requires an additional thoracotomy placed under the robotic arms, so access is somewhat limited. Alternatively, the cannula could be placed through a 12-mm port with purse-string sutures placed either robotically in a port-only approach or directly with a small-access thoracotomy. Either way, surgical access is limited in the event that control of the aorta is lost.

There are various opinions regarding the optimal venous cannulation approach. Some surgeons prefer a single, dual-stage femoral venous cannula, whereas others use bicaval cannulation in an effort to ensure adequate venous drainage, gaining percutaneous assess to the superior vena cava by cannulating the right internal jugular vein. Vacuum or kinetic-assisted venous drainage provides better drainage when using femoral cannulation, thereby avoiding distention of the right side of the heart and improving exposure and maintaining myocardial hypothermia.

\section{Cross-Clamping}

As with port-access mitral surgery, a transthoracic aortic cross-clamp or an endoaortic occlusion balloon can be used to isolate the coronary circulation from the systemic blood flow. ${ }^{14}$ When using a transthoracic cross-clamp in robotic-assisted cases, it should be inserted through a lateral stab wound in the second intercostal space, 8 to $10 \mathrm{~cm}$ posterior to the left robotic arm to avoid internal and external conflict with the robotic arms. The access site for the cardioplegia catheter is controlled with a purse-string suture and can be placed directly through the incision with long instruments, or robotically.

Alternatively, myocardial preservation with endoaortic balloon occlusion avoids managing the aortic puncture site and eliminates potential for internal or external conflict that can occur between the transthoracic cross-clamp and the robotic arms. ${ }^{17}$ It is also valuable in redo procedures, can vent the aorta, and preserves the intrathoracic workspace. Limitations of endoaortic balloon occlusion are distal balloon migration and obstruction of the arch vessels, impaired exposure of the left fibrous trigone with proximal migration on the balloon, and increased complexity of cannulation. Please refer to Wolfe et al $^{14}$ for a more extensive discussion of approaches to aortic occlusion in MIMVR.

\section{Myocardial Protection and Delivery of Cardioplegia}

There are 4 approaches to myocardial preservation including antegrade, retrograde, combined, and cold fibrillatory arrest. All can successfully be applied in robotic-assisted MIMVR, and these are discussed in detail in Aliawadi et al. ${ }^{13}$

\section{Temperature Management}

The use of mild or moderate hypothermia $\left(28^{\circ} \mathrm{C}\right.$ to $\left.33^{\circ} \mathrm{C}\right)$, whereas not necessary in primary operations, may augment myocardial preservation early in the learning curve and may be useful during longer or more complex procedures. The patient should be cooled during redo operations with a patent internal mammary artery.

\section{Exposure and Port Placement: Minithoracotomy Versus Totally Endoscopic Approaches}

For robotic MIMVR, there are 2 basic approaches: minithoracotomy and totally endoscopic. Whereas positioning, prepping, and draping are similar for both of these approaches, incisions and port placement varies considerably, and incorrect placement can hamper safe completion of the operation. In either case, use of a generous working port, or a standard minithoracotomy incision can aid the transition from minimally invasive to robotic approaches.

The minithoracotomy approach (Fig. 2) is favored by a number of surgeons because of its similarity to a nonrobotic right chest approach and because portions of the operation can be done by direct access through the thoracotomy. The camera is placed in the medial aspect of a standard minithoracotomy incision in the fourth intercostal space. Three robotic arms are then added, one in the second or third intercostal space along the anterior axillary line, one in the fifth or sixth intercostal space just posterior to the anterior axillary line, and the third in the fourth or fifth intercostal space between the midclavicular line and the right lateral border of the sternum.

Incisions can be as small as 4 to $5 \mathrm{~cm}$. Soft tissue retractors enhance exposure, and a small rib spreader is sometimes used. Direct access to the ascending aorta is also facilitated for management of the antegrade vent site. 


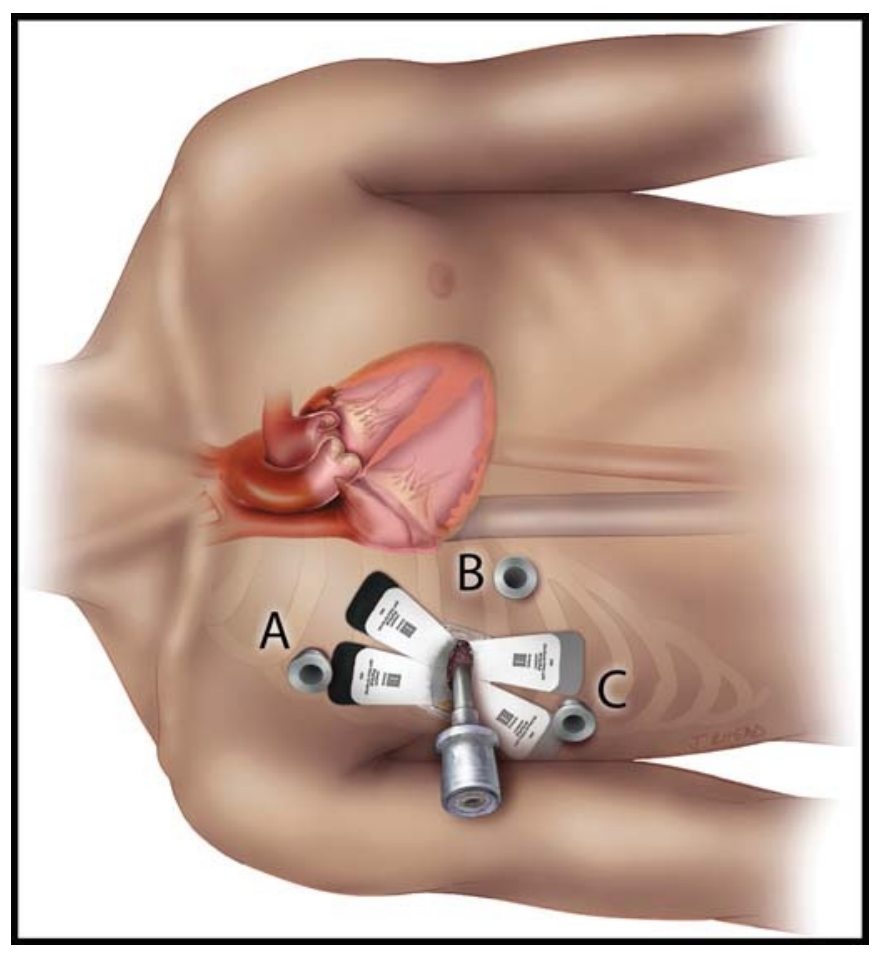

FIGURE 2. Port placement for a robotic-assisted minithoracotomy approach. A, Left robotic port; robotic retractor port (B); right robotic port (C).

The alternative is a totally endoscopic port-access approach (Fig. 3), which is perfectly suited for the roboticassisted MIMVR. The working port can be as small as 15 to $20 \mathrm{~mm}$, and the ports for the robotic arms are $8 \mathrm{~mm}$. The judicious use of $\mathrm{CO}_{2}$ under pressure allows for improved visibility during the extracardiac portions of the procedure, limits the introduction of air during the intracardiac portion, and shortens the time for de-airing. The port-access approach may shorten cross-clamp and pump times and reduce postoperative pain, since there is no rib spreading. Although totally endoscopic approaches are facilitated by the use of the catheter-based balloon occlusion, a transthoracic clamp and robotic placement of an antegrade cardioplegia catheter can be performed.

The basic concept guiding port placement for this approach is to focus the ports toward the apex of a triangle in the left atrium (LA). They should be adequately spaced to provide room for movement of the robotic arms without collision. A 12-mm camera port is routinely placed in the right fourth intercostal space near or just anterior to the anterior axillary line. Depending on the patient's body habitus (deep chest, narrow chest, broad chest, pectus excavatum, etc.), the port may be moved laterally to improve exposure. When the port is placed, the camera is inserted and should be oriented directly at the hilum of the right lung. A 2 -cm working port is then created $2 \mathrm{~cm}$ lateral to the camera port and through the same intercostal space, confirmed by digital palpation. The working port is usually supported with an appropriately sized metal or soft port. Through this port, the tableside surgeon passes the band, follows suture, and removes specimens.

Through the working port, a finger is used to help place the instrument ports, and this, along with gentle $\mathrm{CO}_{2}$ insufflation, will decrease the chance of injury to the lung, diaphragm, or liver. An 8-mm robotic port is placed through the sixth intercostal space anterior to the mid axillary line. Next, an $8-\mathrm{mm}$ port is placed in the second or third intercostal space, between the anterior axillary line and the midclavicular line, directing the ports toward the interatrial groove. The robotic retractor port is placed through the fourth or fifth intercostal space, between the midclavicular line and the right lateral border of the sternum, taking care to avoid injury to the internal mammary artery and vein.

\section{Pericardial Incision and Exposure}

Some robotic surgeons prefer to open the pericardium as far anteriorly as possible, creating a large pericardial flap (Fig. 4). When retracted by sutures to the posterolateral chest wall, the flap holds the lung back and guides instrument exchange into the LA, expediting the introduction of sutures and shortening cross-clamp times. Others prefer to enter the pericardium closer to the pulmonary vein-LA junction and limit dissection. If the latter approach is used, caution is necessary to avoid phrenic nerve injury. Establishing cardiopulmonary bypass can facilitate opening the pericardium.

\section{Atrial Incision}

After cross-clamping and delivery of cardioplegia, the LA is entered at its junction with the right pulmonary veins. There is no advantage to dissecting the intra-atrial groove as in a sternal approach. The oblique sinus is opened to allow extension of the incision further inferiorly to improve exposure.

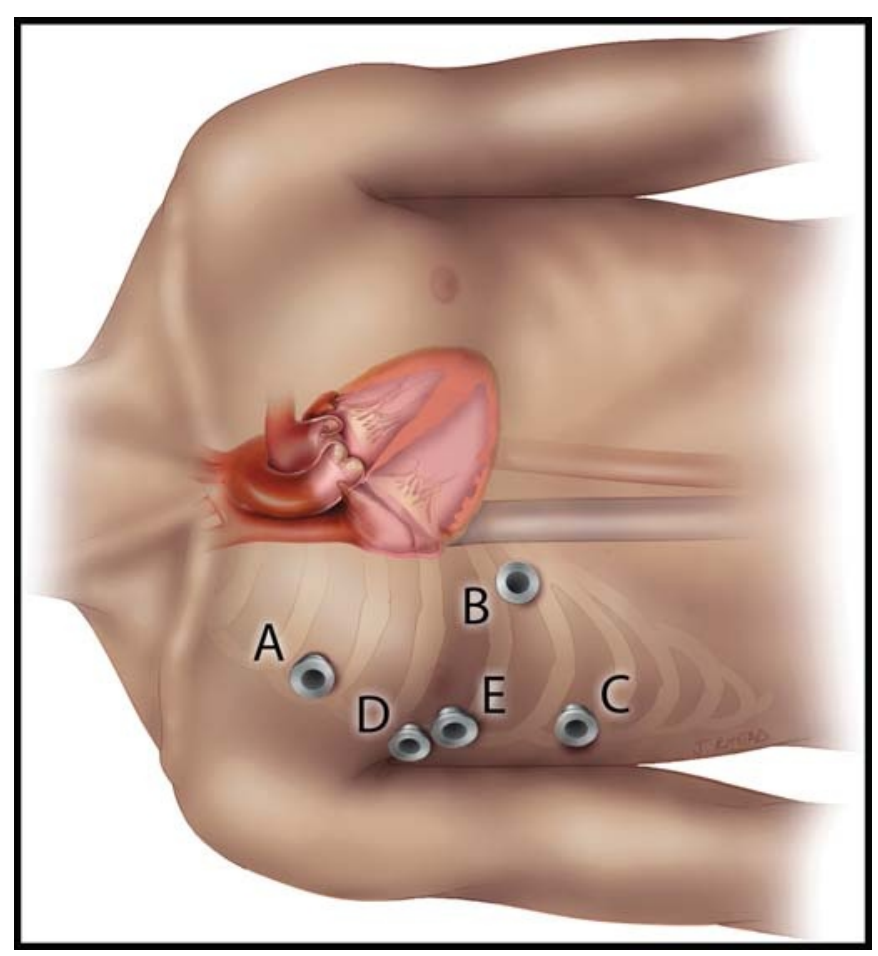

FIGURE 3. Port placement for a totally endoscopic robotic-assisted approach. A, Left robotic port; robotic retractor port (B); right robotic port (C); working port (D); camera port (E). 


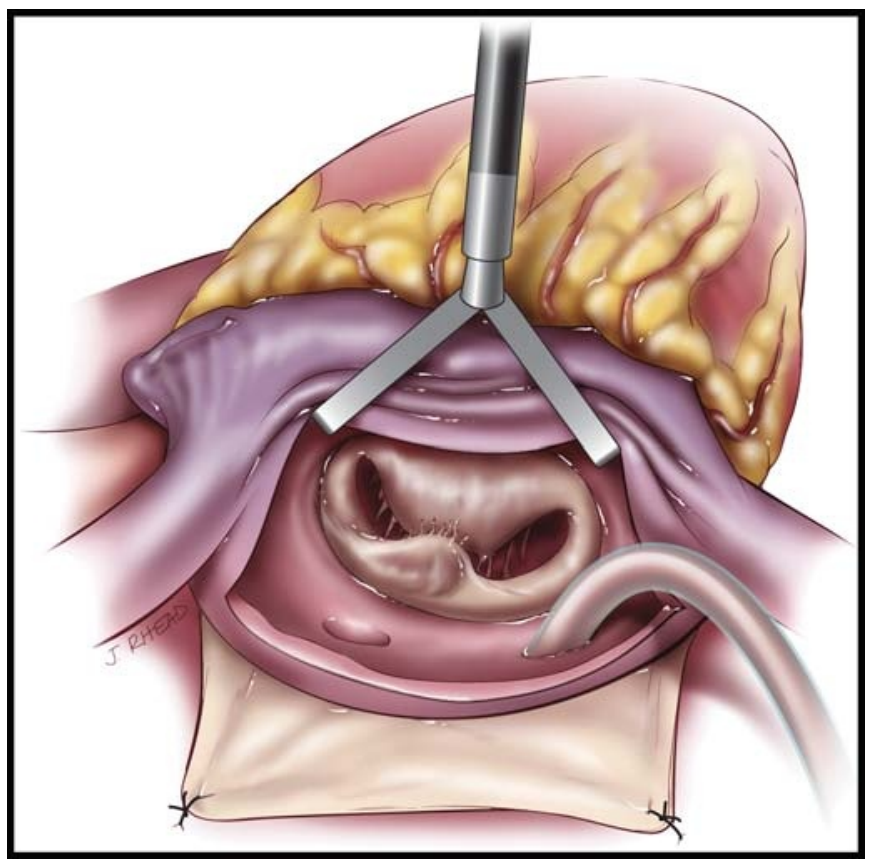

FIGURE 4. Pericardial incision and exposure; MV exposure with dynamic atrial retractor blades deployed.

\section{Intra-atrial Retraction}

The robotic dynamic atrial retractor is inserted through the third-arm port (fifth intercostal space), advanced into the LA under direct camera visualization, and then deployed (Fig. 4). Repositioning is common to facilitate exposure of different parts of the valve during robotic-assisted MIMVR. Closure of the orifice of the left atrial appendage may improve exposure. A sump drain placed in a left pulmonary vein helps to maintain a bloodless field.

\section{Robotic MV Repair}

Valve repair concepts and techniques should follow classic repair techniques, although polytetrafluoroethylene suture is preferred over polypropylene because it is more easily tied with robotic instruments or with a knot pusher than other materials. Use of an autoknotting device to secure braided annuloplasty ring sutures may shorten cross-clamp times compared with tying with robotic instruments. ${ }^{18}$

\section{Robotic MV Replacement}

Robotic-assisted MV replacement may be planned as a primary procedure or used if a repair attempt fails, but it is more challenging than might first be apparent and generally should be performed by more experienced surgeons. A generous working port at least the size of the prosthetic valve is required. Suture management is difficult, and keeping them organized is challenging.

The easiest method for suture management is to place a longitudinal suture guide inferior and lateral to the working port and the robotic ports, in a straight line. Suture placement is begun at the left trigone or approximately the surgeon's 11-o'clock position, with subsequent placement moving in a counter- clockwise fashion. The bedside assistant places the sutures in the suture guide in strict order from left to right. The surgeon then returns to the bedside and, starting at the left trigone and moving counter-clockwise, places the sutures into the valve and then back onto the suture guide to keep them straight. The valve is lowered into view of the robotic camera and then seated carefully. The sutures are then secured. The use of autoknotting devices instead of knot tying can greatly reduce the complexity and increase the speed of this procedure. Left atrial closure is then accomplished with expanded polytetrafluoroethylene (Gore-tex) suture.

\section{De-airing}

De-airing requirements and technique depend on the approach used for exposure and cross-clamping. In general, the port-access, nonthoracotomy, " $\mathrm{CO}_{2}$-tight" approach will introduce less air. The ascending aortic vent and left ventricular vents, placed through the thoracotomy, working port, or in the balloon, are the primary tools for de-airing.

\section{Rhythm Management and Pacing Wires}

While a stable rhythm is usually achieved after unclamping, defibrillation using external patches should be performed if required, but only after removing robotic instruments from the chest and re-expanding the lungs.

With isolated robotic-assisted MIMVR, pacing wires are usually not necessary. If bradycardia persists after chemical manipulation, they can be placed either in the right ventricular free wall or alternatively on the diaphragmatic portion of the left ventricle. Wires are more easily placed before releasing the cross-clamp. When a concurrent tricuspid valve procedure or Cox-maze procedure is performed, pacing wires are frequently necessary.

\section{Closure and Drainage}

Careful inspection of the ports for bleeding will decrease the chance of significant postoperative bleeding. Most groups drain the right pleural space with 1 or 2 tubes, placed through the robotic arm ports. Typically, one tube is advanced high in the pleural space, and the second, usually a soft drain, is positioned in the pericardium. The remaining ports are closed with absorbable suture.

\section{Concurrent Procedures}

Performing concurrent procedures may prolong already extended perfusion and clamp times. We suggest significant experience with primary isolated robotic-assisted MIMVR procedures and consistently shorter clamp times before concurrent procedures are attempted. Surgeons should gain proficiency with simple concurrent procedures such as closure of small atrial septal defects or patent foramen ovale (closed through the LA), pulmonary vein isolation, and closure of the left atrial appendage before moving on to more complex operations such as biatrial Cox-maze procedures. ${ }^{19-21}$ Concurrent tricuspid valve repair, although less common, has been successfully performed by experienced surgeons. ${ }^{22,23}$ 


\section{AVOIDING COMPLICATIONS IN ROBOTIC-ASSISTED MV SURGERY}

\section{Reasons to Convert to Sternotomy}

Early and rapid conversion to a more standard approach when necessary is fundamental to the safe development of a robotic cardiac surgery program. Reasons to convert include but are not limited to those listed below.

\section{Unexpected Right Chest Adhesions}

There is no reliable way to predict the presence or absence of significant adhesions in the right pleural space. If suspected, they may be revealed by inspection through a working port incision, by direct visualization, or through a $5-\mathrm{mm}$ thoracoscope, which can be introduced before formal port placement. Limited medial adhesions can usually be taken down with the robotic instruments. If extensive adhesions are encountered, conversion to either a larger right thoracotomy or, more commonly, a sternotomy is recommended.

\section{Poor Visualization}

If exposure and visualization of the MV is compromised and cannot be improved, then conversion to a sternal approach is warranted.

\section{Extended Time on Cross-Clamp or Bypass}

Robotic-assisted procedures often take longer than open procedures, especially early in a team's learning curve. Designating a team member to impose a hard stop after a predetermined time will allow for safe conversion and completion of the operation. The surgeon should consider clamp times no longer than 2 hours and even shorter in the setting of impaired ventricular function.

\section{Inadequate Myocardial Protection}

Regardless of the myocardial protection strategy, the heart must be rendered electrically quiet, asystolic, and ideally cold when the aorta is clamped. If the surgical team is unable to arrest the heart, conversion should be immediate, although cold fibrillatory arrest could also be considered.

\section{Poor Venous Drainage}

Adequate venous drainage is mandatory. ${ }^{24}$ Inadequate drainage usually originates with improper positioning of the femoral venous cannula and should be remedied before cross-clamping. Helpful options are found in Wolfe et al. ${ }^{14}$ Routine bicaval cannulation and drainage is advisable early in the learning curve.

\section{Bleeding}

Although rare, a potential source of bleeding during robotic-assisted MIMVR is perforation of structuresincluding the superior vena cava, right atrium, right ventricle, aorta, coronary sinus, pulmonary artery, and iliac veinduring line placement, or to any intrathoracic structure during the procedure. Experienced surgeons can sometimes repair these defects robotically, but conversion is always appropriate with these significant operative challenges.

\section{Specific Complications in Robotic-assisted MV Surgery} Unilateral Pulmonary Edema

Clinically significant unilateral pulmonary edema is a rare but life-threatening problem. Its cause is unknown but is likely multifactorial and may result from unfavorable lymphatic drainage, pulmonary artery venting, compromise of bronchial blood flow by the double-lumen endotracheal tube, re-expansion injury, barotrauma, and $\mathrm{CO}_{2}$ pressurization. Most cases are associated with prolonged perfusion and cross-clamp times, and occur early in a team's learning curve. Unilateral pulmonary edema may resolve with supportive care, but extracorporeal membrane oxygenation is occasionally required.

\section{Aortic Dissection}

Dissections have been associated with the endoaortic balloon, transaortic clamp, retrograde perfusion, antegrade cardioplegia needle, and antegrade direct aortic cannulation. ${ }^{25}$ Preoperative computed tomography angiogram of the aorta and run-off through the common femoral artery bifurcation can identify risk factors including occult local dissections. Careful manipulation of guidewires, dilators, catheters, and cannulae minimizes the risk of aortic dissection and other arterial complications. ${ }^{25}$ Please refer to Wolfe et al ${ }^{14}$ for details regarding safe peripheral cannulation.

\section{Compartment Syndrome and Leg Ischemia}

Vascular complications can occur with femoral cannulation. Ischemic injury may lead to compartment syndrome and limb loss. Strategies for minimizing the potential for these problems are discussed in Ailawadi et al. ${ }^{13}$

\section{Nerve Injuries}

Nerves that can be injured during robotic-assisted MIMVR procedures include the phrenic, brachial plexus, and femoral nerve. Phrenic nerve injury can be direct, from traction, or thermal (cold or hot), and is more common in redo operations and in those in which a concurrent Cox-maze procedure is performed. Many will recover within 3 to 6 months if the nerve is not transected. Injury to the brachial plexus is usually minor and can occur from excessive downward traction of the right shoulder while elevating the right hemi-thorax. Injury to the femoral nerve occurs during femoral dissection or when closure sutures are placed too deep lateral to the artery.

\section{Stroke}

Concerns have been raised that MIMVR surgery may have a higher stroke risk than standard surgery. ${ }^{26}$ Use of $\mathrm{CO}_{2}$ throughout the procedure and careful de-airing may prevent air-related cerebrovascular injury. Careful preoperative assessment of the patient and his or her vasculature will identify patients at increased risk. ${ }^{13,27}$ Careful identification and removal of any and all debris from the LA is important.

\section{Postoperative Hemorrhage}

Deliberate inspection of robotic ports minimizes postoperative bleeding. Chest tube output greater than $100 \mathrm{cc} / \mathrm{h}$ is cause for concern. Videoscopic re-exploration can be considered in a stable patient, but early sternotomy, even in the ICU, can be lifesaving if there is any hemodynamic instability, concern for LA suture line disruption, or bleeding from an antegrade aortic cardioplegia cannulation site. It is helpful during thoracoscopic re-exploration to position the patient with the right hemithorax elevated into a more lateral position. The camera and working port should be connected if 2 separate ports were made. A 
thoracoscope should be used to visualize all incisions and suture lines.

\section{Lymphocele}

Various strategies have been devised to prevent lymphocele, including limiting dissection to the anterior walls of the vessels, careful use of electrocautery and clips during dissection, meticulous multilayered closure, and drain placement. Lymphoceles often respond to conservative treatment, but if persistent, surgical re-exploration is indicated.

\section{STEPWISE LEARNING}

For MIMVR programmatic success, it is imperative to develop a stable team and a graduated learning pathway. ${ }^{28}$ Team failure is multifactorial and can result from poor patient selection, inadequate mitral procedural volume, too rapid a progression toward robotics, inconsistent tableside surgical assistance, limited experience with TEE, and inadequate support from the hospital or cardiology department.

A team approach is essential. Each member must learn his or her part of the procedure. It is important to divide each learning curve into a stepwise fashion and start by incorporating key parts of the robotic approach into current, standard approaches before the first robotic-assisted operation. With this in mind, we recommend the following:

- Start by using single-shafted instruments, knot pushers, and suture crimping instruments during standard sternotomy cases.

- Allow anesthesia to place necklines during planned sternotomy cases.

- Learn and gain proficiency with peripheral cannulation and perfusion with TEE guidance. Follow a standard preoperative evaluation to exclude patients with anatomy that makes peripheral perfusion problematic.

- Gain proficiency with MV operations performed through an increasingly smaller thoracotomy to become comfortable with videoscopic exposure of the heart, and to gain further experience with endoscopic instruments and remote perfusion and myocardial preservation.

- Perform robot simulation using nonhuman models, starting with simple plastic models and then working on a porcine heart within a plastic chest model, so that the console and tableside surgeons can develop proficiency with docking and instrument manipulation. If available, training on cadaveric models will add another helpful step in the learning process.

- Bring the entire team (console and tableside surgeons, perfusion, anesthesia, and scrub nurse) to visit and train with other successful robotics programs. Watching an experienced team's approach and then having a proctor oversee the first few cases is indispensable as are return visits after the start of your program. following:

Other important training considerations include the

- Mentorship: There are varied approaches to robotic-assisted MIMVR. A team should learn and adopt an integrated approach from a successful team. Over time, the new team can develop its own tailored approach and a variety of techniques to expand indications to a greater variety of patients and operations.

- Concurrent procedures: These should not be included in a team's early learning curve and are best added after proficiency with simple cases is obtained.

- Outcomes: Critical evaluation of a team's successes and failures is an important part of ensuring success and growth. A registry of cases, outcomes, and modifications to avoid complications is advisable.

- Volume: A program will not flourish without adequate volume. It is difficult to recommend a specific number, but it would seem reasonable that a program should have sufficient caseload of mitral repairs to allow at least 20 robotic-assisted MIMVRs to be performed annually.

- Operative efficiency: Most operative times are increased with minimally invasive procedures and also with most robotic operations, especially early in the learning curve. It is important to track the learning curve by documenting the time required for all steps of the operation and to pursue shorter times.

- Hospital robotic committee: Be prepared to monitor outcomes and know institutional requirements for starting robotic program.

\section{CONCLUSIONS}

As robotic-assisted MIMVR continues to evolve, there remains little consensus among surgeons regarding appropriate training of robotics teams, correct patient selection, and standardization of technical approaches. This group of experienced surgeons attempted to define these parameters and suggested standard approaches to robotic-assisted MIMVR. We have developed recommendations regarding training pathways, patient selection, and technical approaches to robotic-assisted MIMVR, and advised the extension of these techniques to concurrent procedures and to more complex patients.

\section{REFERENCES}

1. Mohr FW, Falk V, Diegeler A, Walther T, van Son JA, Autschbach R. Minimally invasive port-access mitral valve surgery. J Thorac Cardiovasc Surg. 1998;115:567-574.

2. Carpentier A, Loulmet D, Aupècle B, et al. Computer assisted open heart surgery. First case operated on with success [in French]. C R Acad Sci III. 1998;321:437-442.

3. Chitwood WR Jr, Nifong LW, Elbeery JE, et al. Robotic mitral valve repair: trapezoidal resection and prosthetic annuloplasty with the da Vinci surgical system. J Thorac Cardiovasc Surg. 2000;120:1171-1172.

4. Grossi EA, Lapietra A, Applebaum RM, et al. Case report of robotic instrument-enhanced mitral valve surgery. J Thorac Cardiovasc Surg. 2000; 120:1169-1171.

5. Nifong LW, Chitwood WR, Pappas PS, et al. Robotic mitral valve surgery: a United States multicenter trial. J Thorac Cardiovasc Surg. 2005;129:1395-1404.

6. Chitwood WR Jr, Rodriguez E, Chu MW, et al. Robotic mitral valve repairs in 300 patients: a single-center experience. J Thorac Cardiovasc Surg. 2008;136:436-441.

7. Mihaljevic T, Jarrett CM, Gillinov AM, et al. Robotic repair of posterior mitral valve prolapse versus conventional approaches: potential realized. J Thorac Cardiovasc Surg. 2011;141:72-80.

8. Mihaljevic T, Pattakos G, Gillinov AM, et al. Robotic posterior mitral leaflet repair: neochordal versus resectional techniques. Ann Thorac Surg. 2013; 95:787-794.

9. Murphy DA, Miller JS, Langford DA, Snyder AB. Endoscopic robotic mitral valve surgery. J Thorac Cardiovasc Surg. 2006;132:776-781. 
10. Ramzy D, Trento A, Cheng W, et al. Three hundred robotic-assisted mitral valve repairs: the Cedars-Sinai experience. J Thorac Cardiovasc Surg. 2014; 147:228-235.

11. Rodriguez E, Kypson AP, Moten SC, Nifong LW, Chitwood WR Jr. Robotic mitral surgery at East Carolina University: a 6-year experience. Int J Med Robot. 2006;2:211-215.

12. Rodriguez E, Chu MW, Narron J, Gersch K, Nifong LW, Chitwood WR Jr Robotic mitral valve repairs requiring reoperations. Innovations. 2008;3: $12-15$.

13. Ailawadi G, Agnihotri AK, Mehall JR, et al. Minimally invasive mitral valve surgery I: patient selection, evaluation, and planning. Innovations. 2016;11: 243-250.

14. Wolfe JA, Malaisrie SC, Farivar RS, et al. Minimally invasive mitral valve surgery II: surgical technique and postoperative management. Innovations. 2016;11:251-259.

15. Felger JE, Chitwood WR Jr, Nifong LW, Holbert D. Evolution of mitral valve surgery: toward a totally endoscopic approach. Ann Thorac Surg. 2001; 72:1203-1208.

16. Glower DD, Komtebedde J, Clements FM, Debruijn NP, Stafford-Smith M, Newman MF. Direct aortic cannulation for port-access mitral or coronary artery bypass grafting. Ann Thorac Surg. 1999;68:1878-1880.

17. Reichenspurner H, Detter C, Deuse T, Boehm DH, Treede H, Reichart B. Video and robotic-assisted minimally invasive mitral valve surgery: a comparison of the port-access and transthoracic clamp techniques. Ann Thorac Surg. 2005;79:485-490.

18. Mandal K, Alwair H, Nifong WL, Chitwood WR Jr. Robotically assisted minimally invasive mitral valve surgery. $J$ Thorac Dis. 2013;5(Suppl 6): S694-S703.
19. Arcidi JM Jr, Rodriguez E, Elbeery JR, Nifong LW, Efird JT, Chitwood WR Jr. Fifteen-year experience with minimally invasive approach for reoperations involving the mitral valve. J Thorac Cardiovasc Surg. 2012;143:1062-1068.

20. Nifong LW, Rodriguez E, Chitwood WR Jr. 540 consecutive robotic mitral valve repairs including concomitant atrial fibrillation cryoablation. Ann Thorac Surg. 2012;94:38-43.

21. Reade CC, Johnson JO, Bolotin G, et al. Combining robotic mitral valve repair and microwave atrial fibrillation ablation: techniques and initial results. Ann Thorac Surg. 2005;79:480-484.

22. Jones BA, Krueger S, Howell D, Meinecke B, Dunn S. Robotic mitral valve repair: a community hospital experience. Tex Heart Inst J. 2005;32:143-146.

23. Lewis CT, Stephens RL, Tyndal CM, Cline JL. Concomitant robotic mitral and tricuspid valve repair: technique and early experience. Ann Thorac Surg. 2014;97:782-788.

24. Sareyyupoglu B, Suri RM, Rehfeldt KH, Burkhart HM. An unexpected cause of poor venous drainage during robotic mitral valve repair. Interact Cardiovasc Thorac Surg. 2010;10:146-147.

25. Jeanmart H, Casselman FP, De Grieck Y, et al. Avoiding vascular complications during minimally invasive, totally endoscopic intracardiac surgery. J Thorac Cardiovasc Surg. 2007;133:1066-1070.

26. Cheng DC, Martin J, Lal A, et al. Minimally invasive versus conventional open mitral valve surgery: a meta-analysis and systematic review. Innovations. 2011;6:84-103.

27. Crooke GA, Schwartz CF, Ribakove GH, et al. Retrograde arterial perfusion, not incision location, significantly increases the risk of stroke in reoperative mitral valve procedures. Ann Thorac Surg. 2010;89:723-729.

28. Yaffee DW, Loulmet DF, Kelly LA, et al. Can the learning curve of totally endoscopicrobotic mitral valve repair be short-circuited? Innovations. 2014;9:43-48. 